\title{
ANÁLISE DAS DIVERGÊNCIAS MORFOLOGICAS DE HYLA BIPUNCTATA SPIX EM DUAS POPULAÇŐES DO RIO DE JANEIRO E ESPÍRITO SANTO, BRASIL (AMPHIBIA, ANURA, HYLIDAE)
}

\author{
André Gonçalves Dias ${ }^{1}$ \\ Carlos Alberto Gonçalves da Cruz ${ }^{2}$
}

\begin{abstract}
ANALISYS OF THE MORPHOLOGICALS DIVERGENCIES OF Hyla bipunctata SPIX IN TWO POPULATIONS FROM RIO DE JANEIRO AND ESPÍRITO SANTO, BRAZIL (AMPHIBIA, ANURA, HYLIDAE). This paper compares some morphological parameters of Hyla bipunctata Spix, 1824 adults from two populations, one from Itaguaí (Rio de Janeiro) and the other from Linhares (Espírito Santo). Some morphometric data of the morphological variation between those populations are studied.

KEY WORDS. Amphibia, Anura, Hylidae, Hyla bipunctata
\end{abstract}

Os processos de divergência morfológica são devido tanto a variacões de natureza genética quanto às flutuações ambientais a que as diversas formas de uma população estão sujeitas ao longo de uma coexistência temporal (LI, 1955).

Detectar e descrever variações em caracteres quantitativos como meio de discriminação intra e interpopulacional é um dos principais objetivos da morfometria. As medições em porções anatômicas homólogas devem expressar, com boa margem de certeza, fontes de variação que discriminem ou não as formas que compõem uma população.

Foram analisados quantitativamente diversos dos caracteres habitualmente utilizados na sistemática de anfíbios com a finalidade de se interpretar a diferenciação e a divergência morfológica, neles observados, entre uma população de Hyla bipunctata Spix, 1824 do Rio de Janeiro e outra do Espírito Santo.

\section{MATERIAL E MÉTODOS}

Foram utilizados 56 exemplares adultos do sexo masculino e 12 exemplares do sexo feminino em cada uma das populações provenientes de coletas realizadas na Reserva Florestal da Companhia Vale do Rio Doce, município de Linhares (Espírito Santo); no Campus da Universidade Federal Rural do Rio de Janeiro e no Horto Florestal de Santa Cruz, ambas em Seropédica, Itaguaí, Rio de Janeiro.

1) Graduando em Engenharia Agronômica, Universidade Federal Rural do Rio de Janeiro, 23851-970 Itaguaí, Rio de Janeiro, Brasil. Bolsista do CNPq.

2) Departamento de Biologia Animal, Universidade Federal Rural do Rio de Janeiro, 23851-970 Itaguaí, Rio de Janeiro, Brasil. 
O procedimento estatístico se deu por teste de média (teste "t de Student", à nível de 5\% de probabilidade) (FISHER e YATES, 1943), calcado em parâmetros morfológicos coletados com auxilio de paquímetro e microscópio estereoscópico Wild M-5.

Os caracteres comparados morfométricamente em ambos os sexos foram: comprimento rostro-anal, comprimento da tíbia, comprimento do pé, comprimento da cabeça, largura da cabeça, diâmetro ocular e diâmetro timpânico. O comprimento do pé utilizado foi a distância compreendida entre a articulação tíbio-tarsal e a extremidade distal do artelho mais longo.

\section{RESULTADOS}

Os dados processados estatísticamente, bem como sua análise em função de duas hipóteses para as populações, em ambos os sexos, estão expostos nas tabelas I e II, onde constam: médias $(\overline{\mathrm{x}})$, variâncias $\left(\mathrm{S}^{2}\right)$, graus de liberdade (GL), os valores de "t" calculados (tc) e tabelados (tt), as hipóteses de nulidade (Ho) e alternativas $\left(\mathrm{H}_{1}\right)$, rejeição de $\mathrm{Ho}(\mathrm{R})$, aceite de $\mathrm{H}_{0}(\mathrm{~A})$, além dos caracteres analizados.

Tabela I. Caracteres comparados morfométricamente em machos de Hyla bipunctata. Graus de liberdade (GL), "t" calculados (Tc), "t" tabelados (Tt), hipótese de nulidade ( $\left.\mathrm{H}_{0}\right)$, rejeição de $\mathrm{H}_{0}$ (R), aceite de $\mathrm{H}_{0}$ (A), C.R.A. = comprimento rostro-anal, C.tib = comprimento da tíbia, C.pé = comprimento do pé, C.cab. $=$ comprimento da cabeça, L.cab. $=$ largura da cabeça, D.oc. $=$ diâmetro ocular, D.timp. = diâmetro timpânico.

\begin{tabular}{|c|c|c|c|c|c|c|c|c|}
\hline \multirow{2}{*}{ Machos } & \multicolumn{2}{|c|}{ Rio de Janeiro } & \multicolumn{2}{|c|}{ Espírito Santo } & \multirow{2}{*}{ GL } & \multirow{2}{*}{$\mathrm{Tc}$} & \multirow{2}{*}{$\mathrm{Tt}$} & \multirow{2}{*}{$\mathrm{A} / \mathrm{R}: \mathrm{H}_{0}$} \\
\hline & $\bar{X}$ & $\mathrm{~S}^{2}$ & $\bar{x}$ & $\mathrm{~S}^{2}$ & & & & \\
\hline C.R.A. & 22,13 & 1,03 & 26,12 & 1,02 & 55 & 14,77 & 2,00 & $\mathbf{R}$ \\
\hline C.tib. & 12,39 & 0,44 & 14,35 & 0,48 & 55 & 11,31 & 2,00 & $\mathbf{R}$ \\
\hline C.pé. & 16,35 & 0,40 & 19,36 & 0,50 & 55 & 14,55 & 2,00 & $\mathbf{R}$ \\
\hline C.cab. & 7,35 & 0,24 & 8,41 & 0,49 & 55 & 7,50 & 2,00 & $\mathbf{R}$ \\
\hline L.cab. & 7,30 & 0,22 & 8,85 & 0,31 & 55 & 10,96 & 2,00 & $\mathbf{R}$ \\
\hline D.oc. & 2,09 & 0,08 & 2,94 & 0,06 & 55 & 12,02 & 2,00 & $\mathrm{R}$ \\
\hline D.timp. & 1,00 & 0,00 & 1,62 & 0,24 & 55 & 7,75 & 2,00 & $\mathrm{R}$ \\
\hline
\end{tabular}

$\mathrm{H}_{0}: \overline{\mathrm{x}}_{1}=\overline{\mathrm{x}}_{2} ; \mathrm{H}_{1}: \overline{\mathrm{x}}_{1}=\overline{\mathrm{x}}_{2}$

\section{DISCUSSÃO}

$\mathrm{Na}$ análise dos exemplares machos das duas populacões, foram obtidas diferenças estatísticas altamente significativas a nível de $5 \%$ de probabilidade para comprimento rostro-anal, comprimento de tíbia, comprimento de pé, largura de cabeça e diâmetro ocular, e diferenças moderadamente significativas para comprimento de cabeça e diâmetro timpânico. Estas análises levam à rejeição da hipótese de que as populações consideradas sejam estatísticamente homogêneas. 
Tabela II. Caracteres comparados morfométricamente em fêmeas de Hyla bipunctata. Graus de liberdade (GL), "t" calculados (Tc), "t" tabelados (Tt), hipótese de nulidade $\left(\mathrm{H}_{0}\right)$, rejeição de $\mathrm{H}_{0}$ (R), aceite de $\mathrm{H}_{0}(\mathrm{~A})$, C.R.A. = comprimento rostro-anal, C.tib = comprimento da tíbia, C.pé = comprimento do pé, C.cab. $=$ comprimento da cabeça, L.cab. $=$ largura da cabeça, D.oc. $=$ diâmetro ocular, D.timp. = diâmetro timpânico.

\begin{tabular}{|c|c|c|c|c|c|c|c|c|}
\hline \multirow{2}{*}{ Fêmeas } & \multicolumn{2}{|c|}{ Rio de Janeiro } & \multicolumn{2}{|c|}{ Espírito Santo } & \multirow{2}{*}{ GL } & \multirow{2}{*}{$\mathrm{Tc}$} & \multirow{2}{*}{$\mathrm{Tt}$} & \multirow{2}{*}{$\mathrm{A} / \mathrm{R}: \mathrm{H}_{0}$} \\
\hline & $\overline{\mathrm{X}}$ & $s^{2}$ & $\bar{x}$ & $\mathrm{~S}^{2}$ & & & & \\
\hline C.R.A. & 23,14 & 4,49 & 30,17 & 0,96 & 11 & 7,85 & 2,20 & $\mathrm{R}$ \\
\hline C.tib. & 13,29 & 0,59 & 15,83 & 6,55 & 11 & 2,34 & 2,20 & $\mathrm{R}$ \\
\hline C.pé. & 17,00 & 1,00 & 22,00 & 0,79 & 11 & 9,61 & 2,20 & $\mathrm{R}$ \\
\hline C.cab. & 8,00 & 0,34 & 8,67 & 0,27 & 11 & 2,11 & 2,20 & A \\
\hline L.cab. & 7,29 & 3,92 & 10,67 & 0,27 & 11 & 4,33 & 2,20 & $\mathrm{R}$ \\
\hline D.oc. & 2,57 & 0,28 & 3,00 & 0,00 & 11 & 2,15 & 2,20 & A \\
\hline D.timp. & 1,14 & 0,14 & 2,00 & 0,00 & 11 & 6,14 & 2,20 & $\mathrm{R}$ \\
\hline
\end{tabular}

$\mathrm{H}_{0}: \overline{\mathrm{x}}_{1}=\overline{\mathrm{x}}_{2} ; \mathrm{H}_{1}: \overline{\mathrm{x}}_{1}=\overline{\mathrm{x}}_{2} ;$

Para exemplares do sexo feminino, nas duas populações, foram obtidas diferenças estatísticas altamente significativas para caracteres como comprimento rostro-anal, comprimento do pé, largura da cabeça e diâmetro timpânico. Obeservam-se, ainda, diferenças estatísticas significativas em segmentos anatômicos como comprimento da tíbia e relativamente nenhuma diferença estatística significativa em diâmetro ocular e comprimento da cabeça, fato este atribuído, muito provavelmente, ao reduzido número de fêmeas (onze graus de liberdade contra cinqüenta e cinco graus de liberdade para machos), o que se deve, naturalmente, à dificuldade de obtenção de fêmeas no campo. Estes dados não invalidam a hipótese da existência de uma heterogeneidade entre as populações consideradas e para o sexo em questão.

Do ponto de vista das larvas de $H$. bipunctata, a nível populacional, não houve a possibilidade de aplicar-se os referidos testes estatísticos na medida em que não houve disponibilidade de material em número e estágios suficientemente adequados. Porém, calcado em observações puramente visuais verificou-se que na população de larvas de $H$. bipunctata do Espírito Santo ocorriam indíviduos de tamanho reduzido, enquanto que nas larvas do do Rio de Janeiro os indíviduos apresentavam dimensões bem acima das diagnosticadas para aqueles do Espírito Santo.

\section{REFERÊNCIAS BIBLIOGRÁFICAS}

FISHER, R.A. \& F. YATES. 1943. Statistical Tables. Edinburgh, Oliver \& Boyd, 2nd ed., VIII + 98p.

LI, C.C. 1955. Population Genetics. Chicago, Univarsity of Chicago Press, $\mathrm{XI}+366 \mathrm{p}$. 